Bull. Korean Math. Soc. 52 (2015), No. 1, pp. 247-261

http://dx.doi.org/10.4134/BKMS.2015.52.1.247

\title{
ON PROPERTIES RELATED TO REVERSIBLE RINGS
}

\author{
Da Woon Jung, Nam Kyun Kim, Yang Lee, and Sung Ju Ryu
}

Abstract. We study the connections between idempotents and zerodivisors in several kinds of ring theoretic properties. We next study several ring theoretic properties and examples related to reversible rings.

\section{Connections between idempotents and zero-divisors}

Throughout this note every ring is associative with identity unless otherwise stated. Let $R$ be a ring (possibly without identity). Denote the $n$ by $n$ full (resp., upper triangular) matrix ring over $R$ by $\operatorname{Mat}_{n}(R)$ (resp., $U_{n}(R)$ ). Following the literature, we use $D_{n}(R)=\left\{\left(a_{i j}\right) \in U_{n}(R) \mid\right.$ all diagonal entries are equal $\}$. Use $e_{i j}$ for the matrix with $(i, j)$-entry 1 and elsewhere 0 . $\mathbb{Z}\left(\mathbb{Z}_{n}\right)$ denotes the ring of integers (modulo $n$ ). Let $J(R), N_{*}(R), N^{*}(R)$, and $N(R)$ denote the Jacobson radical, the prime radical, the upper nilradical (i.e., sum of all nil ideals), and the set of all nilpotent elements in $R$, respectively. It is well-known that $N^{*}(R) \subseteq J(R)$ and $N_{*}(R) \subseteq N^{*}(R) \subseteq N(R)$. Use $I(R)$ to denote the set of all idempotents in $R$.

A ring is usually called reduced if it has no nonzero nilpotent elements. Due to Lambek [17], a ring $R$ is symmetric if $r s t=0$ implies $r t s=0$ for all $r, s, t \in R$. While, Anderson-Camillo [4] used the term $Z C_{3}$ for symmetric. Commutative rings are clearly symmetric. Reduced rings are symmetric by [4, Theorem I.3], but there are many kinds of non-reduced commutative rings (e.g., $\mathbb{Z}_{n^{l}}$ for $n, l \geq 2)$. Note that a ring $R$ is symmetric if and only if $r_{1} r_{2} \cdots r_{n}=0$ implies $r_{\sigma(1)} r_{\sigma(2)} \cdots r_{\sigma(n)}=0$ for any permutation $\sigma$ of the set $\{1,2, \ldots, n\}$, where $r_{i} \in R$ and $n$ is any positive integer, by [4, Theorem I.1]. Following Cohn [8], a ring $R$ is reversible if $a b=0$ implies $b a=0$ for $a, b \in R$. Anderson-Camillo [4] used the term $Z C_{2}$ for reversible. Symmetric rings are clearly reversible, but the converse need not hold by [4, Example I.5] or Marks [18, Examples 5 and 7]. Following to Bell [7], a ring $R$ is said to satisfy the Insertion-of-FactorsProperty (simply, an IFP ring) if $a b=0$ implies $a R b=0$ for $a, b \in R$. It is easily checked that reversible rings are IFP. A ring is usually called Abelian if every idempotent is central. IFP rings are Abelian rings by [21, Lemma 2.7]

Received December 30, 2013; Revised July 9, 2014.

2010 Mathematics Subject Classification. 16U80, 16D25.

Key words and phrases. reversible ring, idempotent, zero-divisor. 
but the converse fail in general. It is well-known that $N_{*}(R)=N^{*}(R)=N(R)$ for an IFP ring $R$. We will use freely the facts above.

We first connect idempotents with zero-divisors in reduced rings.

Proposition 1.1. For a ring $R$ the following conditions are equivalent:

(1) $R$ is reduced;

(2) $a^{2} \in I(R)$ implies $a=a^{3}$ for $a \in R$.

Proof. (1) $\Rightarrow(2)$. Let $R$ be reduced. Assume $a^{2} \in I(R)$ for $a \in R$. Then $a^{2}\left(1-a^{2}\right)=0$ implies $\left(a\left(1-a^{2}\right)\right)^{2}=0$ since $R$ is reduced, entailing $a\left(1-a^{2}\right)=0$. So $a=a^{3}$.

$(2) \Rightarrow(1)$. Assume the condition (2). Let $a^{2}=0$ for $a \in R$. Then $a=a^{3}$ by the condition, so $a=a^{3}=0$.

Proposition 1.2. For a ring $R$ the following conditions are equivalent:

(1) $R$ is a reduced ring of characteristic 2 ;

(2) $a^{2} \in I(R)$ implies $a \in I(R)$ for $a \in R$.

Proof. $(1) \Rightarrow(2)$. Let $R$ be a reduced ring of characteristic 2. Assume $a^{2} \in I(R)$ for $a \in R$. Then $a^{2}\left(1-a^{2}\right)=0$. But since $R$ is of characteristic 2 , we get

$$
0=a^{2}\left(1-a^{2}\right)=a^{2}(1-a)(1+a)=a^{2}(1-a)(1-a)=a^{2}(1-a)^{2} .
$$

Since $R$ is reduced, we have $(a(1-a))^{2}=0$, entailing $a(1-a)=0$. So $a=a^{2}$.

$(2) \Rightarrow(1)$. Assume the condition (2). Let $a^{2}=0$ for $a \in R$. Then $a=a^{2}$ by the condition, so $a=0$. Assume on the contrary that the characteristic of $R$ is not 2 .

If the characteristic of $R$ is zero, then $\mathbb{Z} \subseteq R$ and $(-1)^{2}=1 \in I(R)$ but $-1 \notin I(R)$, a contradiction to the condition $(2)$.

Next if the characteristic of $R$ is $n \geq 3$, then $\mathbb{Z}_{n} \subseteq R$ and $(n-1)^{2}=(-1)^{2}=$ $1 \in I(R)$ but $n-1 \notin I(R)$, a contradiction to the condition (2).

The following argument elaborates Proposition 1.2.

Remark 1.3. (1) Let $R$ be a ring of any characteristic and $a \in R$. Assume that $R$ satisfies the condition that $a^{2} \in I(R)$ implies $a \in I(R)$. Then $R$ being reduced can be shown by Proposition 1.1.

(2) If the characteristic of a ring $R$ is a prime $p \geq 3$, then $(1+a)^{p}=1+a$ for any $a \in R$ by help of [13, Exercises 3.1.10(e)].

We next connect idempotents with zero-divisors in reversible rings.

Proposition 1.4. For a ring $R$ the following conditions are equivalent:

(1) $R$ is reversible;

(2) $a b \in I(R)$ implies $b a \in I(R)$ for $a, b \in R$.

Proof. $(1) \Rightarrow(2)$. Let $R$ be reversible. Assume $a b \in I(R)$ for $a, b \in R$. Then $a b(1-a b)=0$. But since $R$ is reversible, we get $0=b(1-a b) a=b a-b a b a$. So $b a \in I(R)$. 
$(2) \Rightarrow(1)$. Assume the condition (2). Let $a b=0$. Then $b a \in I(R)$ by the condition, so $b a=b a b a=0$.

Corollary 1.5. Let $R$ be a reversible ring. If $a b \in I(R)$ for $a, b \in R$, then $a b=b a$.

Proof. Let $R$ be a reversible ring and assume that $a b \in I(R)$ for $a, b \in R$. Then $b a \in I(R)$ by Proposition 1.4. Then we have

$$
b a=b a b a=b(a b) a=(a b) b a=a b(b a)=a(b a) b=a b a b=a b
$$

since $R$ is Abelian.

We next connect idempotents with zero-divisors in symmetric rings.

Proposition 1.6. For a ring $R$ the following conditions are equivalent:

(1) $R$ is symmetric;

(2) $a b c \in I(R)$ implies $a c b=a c b c a b$ for $a, b, c \in R$;

(3) $a b c \in I(R)$ implies acb = acbbca for $a, b, c \in R$;

(4) $a b c \in I(R)$ implies $a c b=a c b a b c$ for $a, b, c \in R$.

Proof. $(1) \Rightarrow(2)$. Let $R$ be symmetric. Assume $a b c \in I(R)$ for $a, b, c \in R$. Then $a b c=a b c a b c$ yields $a b(1-c a b) c=0$. But since $R$ is symmetric, we get $a c b(1-c a b)=0$, entailing $a c b=a c b c a b$.

$(2) \Rightarrow(1)$. Assume the condition (2). We first show that $R$ is reversible. Let $d e=0$ for $d, e \in R$. Then

$$
e d=1 e d=1 e d e 1 d=0
$$

by the condition, entailing that $R$ is reversible. Next assume that $a b c=0$ for $a, b, c \in R$. Then $a c b=a c b c a b$ by the condition. But $a b c=0$ yields $a c b c a b=0$ since $R$ is IFP.

The equivalences of the conditions (2), (3), and (4) are shown by help of Corollary 1.5, noting that $a b c=b c a=c a b$ whenever $a b c \in I(R)$.

Let $R$ be a ring and $a, b, c \in R$. Assume that $R$ satisfies the condition that $a b c \in I(R)$ implies $a c b \in I(R)$. Then $R$ is symmetric by a similar method to the proof of Proposition 1.6. However we do not know whether symmetric rings yield the condition.

Question. Does a symmetric ring $R$ satisfy the condition that $a b c \in I(R)$ implies $a c b \in I(R)$ for $a, b, c \in R$ ?

Proposition 1.7. For a ring $R$ the following conditions are equivalent:

(1) $R$ is IFP;

(2) For $a, b \in R, a b \in I(R)$ implies arb = arbab for all $r \in R$.

Proof. $(1) \Rightarrow(2)$. Let $R$ be a IFP ring. Assume $a b \in I(R)$ for $a, b \in R$. Then $a b(1-a b)=0$ yields $a b r(1-a b)=0$ for all $r \in R$ since $R$ is IFP. So $a r b=a r b a b$ for all $r \in R$. 
$(2) \Rightarrow(1)$. Assume the condition (2). Let $a b=0$ for $a, b \in R$. Then for all $r \in R$ arb $=a r b a b=0$ by the condition.

Proposition 1.8. Let $R$ be a ring. Assume that $R$ satisfies the condition that $a b \in I(R)$ implies arb $\in I(R)$ for all $r \in R$, where $a, b \in R$. Then $R$ is IFP.

Proof. Let $a b=0$ for $a, b \in R$. Then, by assumption, $a r b \in I(R)$ for all $r \in R$. Moreover, $(\text { barbar })^{2}=b($ arbarbarb)ar $=b($ arb)ar, so barbar $\in I(R)$. Thus $a r b=a r b a r b a r b=a r($ barbar $) b=a($ barbar $) r b=0$ since $R$ is Abelian.

The converse of Proposition 1.8 need not hold. Let $R$ be a reduced ring. Then $D_{3}(R)$ is IFP by [16, Proposition 1.2]. And $\left(e_{11}+e_{22}+e_{33}\right)\left(e_{11}+e_{22}+\right.$ $\left.e_{33}\right)=e_{11}+e_{22}+e_{33} \in I\left(D_{3}(R)\right)$ but $\left(e_{11}+e_{22}+e_{33}\right) e_{13}\left(e_{11}+e_{22}+e_{33}\right)=$ $e_{13} \notin I\left(D_{3}(R)\right)$.

\section{Related concepts and examples}

Symmetric rings play an important role in noncommutative ring theory as well as reversible rings. The concept of symmetric rings was introduced by Lambek [17] to unify sheaf representations of commutative rings and reduced rings. Prior to Cohn's work, reversible rings were studied under the names completely reflexive and zero commutative by Mason [19] and Habeb [11], respectively. Tuganbaev [22] investigated reversible rings in his monograph on distributive lattices arising in ring theory, using the name commutative at zero in place of reversible. Recently, various generalized conditions of symmetric and reversible rings have studied by many authors, and the results obtained here were applied to many sorts of problems arising in noncommutative ring theory. In this section, of particular interest will be central symmetric and central reversible rings. We continue the study of Kafkas et al. [14], providing more results for the structure. Thus this work can also provide a sort of bridge between commutative and noncommutative ring theory.

Following Kafkas et al. [14], a ring $R$ is called central symmetric if for any $a, b, c \in R, a b c=0$ implies $b a c$ belongs to the center of $R$. Commutative rings, reduced rings and symmetric rings are clearly central symmetric. One may suspect that central symmetric ring property is left right symmetric. However we answer this question negatively in the following two examples. Use $Z(R)$ to denote the set of all centers in $R$.

Example 2.1. Let $\mathbb{Z}_{2}$ be the field of integers modulo 2 and $A=\mathbb{Z}_{2}\langle a, b\rangle$ be the free algebra generated by noncommuting indeterminates $a, b$ over $\mathbb{Z}_{2}$. Let $I$ be the ideal of $A$ generated by

$$
a^{2} b, b^{2} a, a b a b, b a b a \text { and } r_{1} r_{2} r_{3} r_{4} r_{5},
$$

where $r_{1}, r_{2}, r_{3}, r_{4}, r_{5} \in R$ and set $R=A / I$. We identity every element of $A$ with its image in $R$ for simplicity. We have $a a b=0$, but $(a b a) a \neq 0$ and $a(a b a)=0$, entailing $a b a \notin Z(R)$; hence $R$ is not the right version of central symmetric. 
We will show that $R$ is central symmetric. Let $\alpha, \beta, \gamma \in R$. Then $\alpha, \beta$ and $\gamma$ can be express as in the following forms:

$$
\begin{aligned}
& \alpha=\alpha_{0}+f_{1}(a)+g_{1}(b)+a g_{2}(b)+b f_{2}(a)+a b f_{3}(a)+b a g_{3}(b), \\
& \beta=\beta_{0}+h_{1}(a)+k_{1}(b)+a k_{2}(b)+b h_{2}(a)+a b h_{3}(a)+b a k_{3}(b)
\end{aligned}
$$

and $\quad \gamma=\gamma_{0}+l_{1}(a)+m_{1}(b)+a m_{2}(b)+b l_{2}(a)+a b l_{3}(a)+b a m_{3}(b)$,

where $\alpha_{0}, \beta_{0}, \gamma_{0} \in \mathbb{Z}_{2}, f_{i}(x), g_{i}(x), h_{i}(x), k_{i}(x), l_{i}(x), m_{i}(x) \in \mathbb{Z}_{2}(x)$ for all $i, j$ and the constant terms of $f_{i}(x), g_{i}(x), h_{i}(x), k_{i}(x), l_{i}(x), m_{i}(x)$ are all zero. Now suppose $\alpha \beta \gamma=0$. Then

$$
\begin{aligned}
0= & \alpha \beta \gamma \\
= & \alpha_{0} \beta_{0} \gamma_{0}+\left\{\alpha_{0} \beta_{0} l_{1}(a)+\alpha_{0} \gamma_{0} h_{1}(a)+\beta_{0} \gamma_{0} f_{1}(a)+\alpha_{0} h_{1}(a) l_{1}(a)\right. \\
& \left.+\beta_{0} f_{1}(a) l_{1}(a)+\gamma_{0} f_{1}(a) h_{1}(a)+f_{1}(a) h_{1}(a) l_{1}(a)\right\}+\left\{\alpha_{0} \beta_{0} m_{1}(b)\right. \\
& +\alpha_{0} \gamma_{0} k_{1}(b)+\beta_{0} \gamma_{0} g_{1}(b)+\alpha_{0} k_{1}(b) m_{1}(b)+\beta_{0} g_{1}(b) m_{1}(b)+\gamma_{0} g_{1}(b) k_{1}(b) \\
& \left.+g_{1}(b) k_{1}(b) m_{1}(b)\right\}+\left\{\alpha_{0} \beta_{0} a m_{2}(b)+\alpha_{0} \gamma_{0} a k_{2}(b)+\beta_{0} \gamma_{0} a g_{2}(b)\right. \\
& +\alpha_{0} h_{1}(a) m_{1}(b)+\alpha_{0} a k_{2}(b) m_{1}(b)+\beta_{0} f_{1}(a) m_{1}(b)+\beta_{0} a g_{2}(b) m_{1}(b) \\
& \left.+\gamma_{0} f_{1}(a) k_{1}(b)+\gamma_{0} a g_{2}(b) k_{1}(b)+f_{1}(a) k_{1}(b) m_{1}(b)+a g_{2}(b) k_{1}(b) m_{1}(b)\right\} \\
& +\left\{\alpha_{0} \beta_{0} b l_{2}(a)+\alpha_{0} \gamma_{0} b h_{2}(a)+\beta_{0} \gamma_{0} b f_{2}(a)+\alpha_{0} k_{1}(b) l_{1}(a)+\alpha_{0} b h_{2}(a) l_{1}(a)\right. \\
& +\beta_{0} g_{1}(b) l_{1}(a)+\beta_{0} b f_{2}(a) l_{1}(a)+\gamma_{0} g_{1}(b) h_{1}(a)+\gamma_{0} b f_{2}(a) h_{1}(a) \\
& \left.+g_{1}(b) h_{1}(a) l_{1}(a)+b f_{2}(a) h_{1}(a) l_{1}(a)\right\}+\left\{\alpha_{0} \beta_{0} a b l_{3}(a)+\alpha_{0} \gamma_{0} a b h_{3}(a)\right. \\
& +\beta_{0} \gamma_{0} a b f_{3}(a)+\alpha_{0} h_{1}(a) b l_{2}(a)+\alpha_{0} a k_{2}(b) l_{1}(a)+\alpha_{0} a b h_{3}(a) l_{1}(a) \\
& +\beta_{0} f_{1}(a) b l_{2}(a)+\beta_{0} a g_{2}(b) l_{1}(a)+\beta_{0} a b f_{3}(a) l_{1}(a)+\gamma_{0} f_{1}(a) b h_{2}(a) \\
& +\gamma_{0} a g_{2}(b) h_{1}(a)+\gamma_{0} a b f_{3}(a) h_{1}(a)+f_{1}(a) k_{1}(b) l_{1}(a)+f_{1}(a) b h_{2}(a) l_{1}(a) \\
& \left.+a g_{2}(b) h_{1}(a) l_{1}(a)\right\}+\left\{\alpha_{0} \beta_{0} b a m_{3}(b)+\alpha_{0} \gamma_{0} b a k_{3}(b)+\beta_{0} \gamma_{0} b a g_{3}(b)\right. \\
& +\alpha_{0} k_{1}(b) a m_{2}(b)+\alpha_{0} b h_{2}(a) m_{1}(b)+\alpha_{0} b a k_{3}(b) m_{1}(b)+\beta_{0} g_{1}(b) a m_{2}(b) \\
& +\beta_{0} b f_{2}(a) m_{1}(b)+\beta_{0} b a g_{3}(b) m_{1}(b)+\gamma_{0} g_{1}(b) a k_{2}(b)+\gamma_{0} b f_{2}(a) k_{1}(b) \\
& +\gamma_{0} b a g_{3}(b) k_{1}(b)+g_{1}(b) h_{1}(a) m_{1}(b)+g_{1}(b) a k_{2}(b) m_{1}(b) \\
& \left.+b f_{2}(a) k_{1}(b) m_{1}(b)\right\} .
\end{aligned}
$$

So we obtain $\alpha_{0}=0, \beta_{0}=0$ or $\gamma_{0}=0$ since $\alpha_{0} \beta_{0} \gamma_{0}$ is unique in the expansion of $\alpha \beta \gamma$.

If $\alpha_{0}=0, \beta_{0}=\gamma_{0}=1$, then $f_{1}(a)+f_{1}(a) l_{1}(a)+h_{1}(a) f_{1}(a)+h_{1}(a) f_{1}(a) l_{1}(a)$ $=0$, entailing $f_{1}(a)=0$. Similarly, $g_{1}(b)=b f_{2}(a)=a g_{2}(b)=a b f_{3}(a)=$ $\operatorname{bag}_{3}(b)=0$. Thus $\alpha=0$. Similarly, if $\beta_{0}=0, \alpha_{0}=\gamma_{0}=1$, then $\beta=0$. Also, if $\gamma_{0}=0, \alpha_{0}=\beta_{0}=1$, then $\gamma=0$

Case 1. $\alpha_{0}=\beta_{0}=0$ and $\gamma_{0}=1$.

We have

$$
\begin{aligned}
& f_{1}(a) h_{1}(a)+f_{1}(a) h_{1}(a) l_{1}(a)=0 \\
& g_{1}(b) k_{1}(b)+g_{1}(b) k_{1}(b) m_{1}(b)=0
\end{aligned}
$$




$$
\begin{aligned}
& a k_{1}(b)+a g_{2}(b) k_{1}(b)+a k_{1}(b) m_{1}(b)+a g_{2}(b) k_{1}(b) m_{1}(b)=0 ; \\
& b h_{1}(a)+b f_{2}(a) h_{1}(a)+b h_{1}(a) l_{1}(a)+b f_{2}(a) h_{1}(a) l_{1}(a)=0 ; \\
& a b h_{2}(a)+a b h_{1}(a)+a b f_{3}(a) h_{1}(a)+a b l_{1}(a)+a b h_{2}(a) l_{1}(a)+a b h_{1}(a) l_{1}(a)=0 ; \\
& b a k_{2}(b)+b a k_{1}(b)+b a g_{3}(b) k_{1}(b)+b a m_{1}(b)+b a k_{2}(b) m_{1}(b)+b a k_{1}(b) m_{1}(b)=0 . \\
& \text { Then } k_{1}(b)=h_{1}(a)=b h_{2}(a)=l_{1}(a)=a k_{2}(b)=m_{1}(b)=0 . \text { Thus } \beta \alpha \gamma= \\
& a b h_{3}(a) f_{1}(a)+b a k_{3}(b) g_{1}(b) \in Z(R) .
\end{aligned}
$$

Case 2. $\beta_{0}=\gamma_{0}=0$ and $\alpha_{0}=1$.

We have

$$
\begin{gathered}
h_{1}(a) l_{1}(a)+f_{1}(a) h_{1}(a) l_{1}(a)=0 ; \\
k_{1}(b) m_{1}(b)+g_{1}(b) k_{1}(b) m_{1}(b)=0 ; \\
a m_{1}(b)+a k_{2}(b) m_{1}(b)+a k_{1}(b) m_{1}(b)+a g_{2}(b) k_{1}(b) m_{1}(b)=0 ; \\
b l_{1}(a)+b h_{2}(a) l_{1}(a)+b h_{1}(a) l_{1}(a)+b f_{2}(a) h_{1}(a) l_{1}(a)=0 ; \\
a b l_{2}(a)+a b l_{1}(a)+a b h_{3}(a) l_{1}(a)+a b l_{1}(a)+a b h_{2}(a) l_{1}(a)+a b h_{1}(a) l_{1}(a)=0 ; \\
b a m_{2}(b)+b a m_{1}(b)+b a k_{3}(b) m_{1}(b)+b a m_{1}(b)+b a k_{2}(b) m_{1}(b)+b a k_{1}(b) m_{1}(b)=0 .
\end{gathered}
$$$$
\text { Then } m_{1}(b)=l_{1}(a)=b l_{2}(a)=a m_{2}(b)=0 \text {. Thus } \beta \alpha \gamma=0 \text {. }
$$

Case 3. $\alpha_{0}=\gamma_{0}=0$ and $\beta_{0}=1$.

We get $\beta \alpha \gamma=0$ by a similar to the computation in Case 2 .

Case 4. $\alpha_{0}=0, \beta_{0}=0$ and $\gamma_{0}=0$.

We have

$$
\begin{gathered}
f_{1}(a) h_{1}(a) l_{1}(a)=g_{1}(b) k_{1}(b) m_{1}(b)=0 ; \\
a k_{1}(b) m_{1}(b)+a g_{2}(b) k_{1}(b) m_{1}(b)=0 ; \\
b h_{1}(a) l_{1}(a)+b f_{2}(a) h_{1}(a) l_{1}(a)=0 ; \\
a b l_{1}(a)+a b h_{2}(a) l_{1}(a)+a b h_{1}(a) l_{1}(a)=0 ; \\
b a m_{1}(b)+b a k_{2}(b) m_{1}(b)+b a k_{1}(b) m_{1}(b)=0 .
\end{gathered}
$$

Then $l_{1}(a)$ and $m_{1}(b)=0$. Thus $\beta \alpha \gamma=0$.

Summarizing, we now have $\beta \alpha \gamma \in Z(R)$ in any case, concluding that $R$ is central symmetric.

Example 2.2. Let $\mathbb{Z}_{2}$ be the field of integers modulo 2 and $A=\mathbb{Z}_{2}\langle a, b\rangle$ be the free algebra generated by the noncommuting indeterminates $a, b$ over $\mathbb{Z}_{2}$. Let $I$ be the ideal of $A$ generated by

$$
a b^{2}, b a^{2}, a b a b, b a b a \text { and } r_{1} r_{2} r_{3} r_{4} r_{5},
$$

where $r_{1}, r_{2}, r_{3}, r_{4}, r_{5} \in R$ and set $R=A / I$. We identity every element of $A$ with its image in $R$ for simplicity. We have $a b b=0$, but $b(b a b) \neq 0$ and $(b a b) b=0$, entailing $b a b \notin Z(R)$; hence $R$ is not a central symmetric ring. However $R$ is the right version of central symmetric by a similar method to the computation in Example 2.1. 
Due to Examples 2.1 and 2.2, a ring $R$ are will be called right central symmetric if $a b c=0$ implies $a c b \in Z(R)$; and left central symmetric if $a b c=0$ implies $b a c \in Z(R)$. It is also obviously that commutative rings, reduced rings and symmetric rings are both left and right central symmetric. As a generalization of reduced rings, a ring is called central reduced [1] if every nilpotent element is central. Central reduced rings are left central symmetric by [14, Lemma 2.5]. We can obtain that central reduced rings are also right central symmetric by applying the method of [14, Lemma 2.5].

Lemma 2.3. If a ring $R$ is a central reduced ring, then it is right central symmetric.

Proof. We apply the proof of [14, Lemma 2.5]. Suppose $a b c=0$ for $a, b, c \in R$. Then, for all $r \in R,(b c r a)^{2}=(b c r a)(b c r a)=0$ so bcra is central since $R$ is central reduced. And $(c r a s b)^{2}=(c r a s b)(c r a s b)=c r a s(b c r a) s b=c r a(b c r a) s s b=$ 0 and hence $c r a s b \in Z(R)$ for any $r, s \in R$. So $(a c b)^{4}=a(c b a c b) a(c b a c b)=$ $a a(c b a c b)^{2}=0$ and thus $a c b$ is central.

Let $R$ be a right central symmetric ring and $a, b, c \in R$. Then we have the following: (1) $a b c=0 \Rightarrow a c b \in Z(R) ;(2) 1 a(b c)=a b c=0 \Rightarrow b c a \in Z(R)$, so $b c b c a=b c a b c=0$; and (3) $1(a b) c=a b c=0 \Rightarrow c a b \in Z(R)$, so $c c a b=c a b c=0$.

A ring $R$ is called central reversible if for any $a, b \in R, a b=0$ implies $b a$ is central in $R$ by [14]. Clearly left or right central symmetric rings are central reversible for rings with identity but the converse need not hold by $[14$, Example 2.8]. Also reversible rings are obviously central reversible. We now give an example to show that there exists a central reversible ring which is not a reversible ring.

Example 2.4. Let $R$ be a commutative and reduced ring. Note that $Z\left(D_{3}(R)\right)$ $=\left\{\left(\begin{array}{ccc}a & 0 & \alpha \\ 0 & a & 0 \\ 0 & 0 & a\end{array}\right) \mid a, \alpha \in R\right\}$. Next, suppose that $A B C=0$ for $A=\left(\begin{array}{ccc}a_{1} & b_{1} & c_{1} \\ 0 & a_{1} & d_{1} \\ 0 & 0 & a_{1}\end{array}\right)$, $B=\left(\begin{array}{ccc}a_{2} & b_{2} & c_{2} \\ 0 & a_{2} & d_{2} \\ 0 & 0 & a_{2}\end{array}\right)$ and $C=\left(\begin{array}{ccc}a_{3} & b_{3} & c_{3} \\ 0 & a_{3} & d_{3} \\ 0 & 0 & a_{3}\end{array}\right) \in D_{3}(R)$. Then $a_{1} a_{3} a_{2}=a_{1} a_{3} b_{2}+$ $a_{1} b_{3} a_{2}+b_{1} a_{3} a_{2}=a_{1} a_{3} d_{2}+a_{1} d_{3} a_{2}+d_{1} a_{3} a_{2}=0$. So $A C B=\left(\begin{array}{lll}0 & 0 & \beta \\ 0 & 0 & 0 \\ 0 & 0 & 0\end{array}\right) \in$ $Z\left(D_{3}(R)\right)$, where $\beta=a_{1} a_{3} c_{2}+a_{1} b_{3} d_{2}+b_{1} a_{3} d_{2}+a_{1} c_{3} a_{2}+b_{1} d_{3} a_{2}+c_{1} a_{3} a_{2}$. Thus $D_{3}(R)$ is right central symmetric and so central reversible. Since $e_{23} e_{12}=0$ but $e_{12} e_{23}=e_{13} \neq 0, D_{3}(R)$ is not reversible and hence is not symmetric.

Above example also means that right central symmetric need not be symmetric. However central reversible and IFP are independent of each other by the following.

Example 2.5. Let $K$ be a field and $R=K\langle a, b, c \mid a b, b a c-c b a, a r a, b r b, c r c\rangle$ for all $r \in R$. Then, clearly $R$ is not IFP. We claim $R$ is right central symmetric. Note that every element of $R$ can be written uniquely in the form $\alpha_{0}+\alpha_{1} a+$ $\alpha_{2} b+\alpha_{3} c+\alpha_{4} a c+\alpha_{5} b a+\alpha_{6} b c+\alpha_{7} c a+\alpha_{8} c b+\alpha_{9} a c b+\alpha_{10} b c a+\alpha_{11} c b a, \alpha_{i} \in K$. 
Let $f g h=0$ with $f=\alpha_{0}+\alpha_{1} a+\alpha_{2} b+\alpha_{3} c+\alpha_{4} a c+\alpha_{5} b a+\alpha_{6} b c+\alpha_{7} c a+$ $\alpha_{8} c b+\alpha_{9} a c b+\alpha_{10} b c a+\alpha_{11} c b a, g=\beta_{0}+\beta_{1} a+\beta_{2} b+\beta_{3} c+\beta_{4} a c+\beta_{5} b a+\beta_{6} b c+$ $\beta_{7} c a+\beta_{8} c b+\beta_{9} a c b+\beta_{10} b c a+\beta_{11} c b a$, and $h=\gamma_{0}+\gamma_{1} a+\gamma_{2} b+\gamma_{3} c+\gamma_{4} a c+$ $\gamma_{5} b a+\gamma_{6} b c+\gamma_{7} c a+\gamma_{8} c b+\gamma_{9} a c b+\gamma_{10} b c a+\gamma_{11} c b a$. Then we have the following system of equalities:

(0) $\quad \alpha_{0} \beta_{0} \gamma_{0}=0$

(1) $\quad \alpha_{1} \beta_{0} \gamma_{0}+\alpha_{0} \beta_{1} \gamma_{0}+\alpha_{0} \beta_{0} \gamma_{1}=0$

(2) $\quad \alpha_{2} \beta_{0} \gamma_{0}+\alpha_{0} \beta_{2} \gamma_{0}+\alpha_{0} \beta_{0} \gamma_{2}=0$;

(3) $\quad \alpha_{3} \beta_{0} \gamma_{0}+\alpha_{0} \beta_{3} \gamma_{0}+\alpha_{0} \beta_{0} \gamma_{3}=0$;

(4) $\quad \alpha_{4} \beta_{0} \gamma_{0}+\alpha_{0} \beta_{4} \gamma_{0}+\alpha_{0} \beta_{0} \gamma_{4}+\alpha_{1} \beta_{3} \gamma_{0}+\alpha_{1} \beta_{0} \gamma_{3}+\alpha_{0} \beta_{1} \gamma_{3}=0$;

(5) $\quad \alpha_{5} \beta_{0} \gamma_{0}+\alpha_{0} \beta_{5} \gamma_{0}+\alpha_{0} \beta_{0} \gamma_{5}+\alpha_{2} \beta_{1} \gamma_{0}+\alpha_{2} \beta_{0} \gamma_{1}+\alpha_{0} \beta_{2} \gamma_{1}=0$;

(6) $\quad \alpha_{6} \beta_{0} \gamma_{0}+\alpha_{0} \beta_{6} \gamma_{0}+\alpha_{0} \beta_{0} \gamma_{6}+\alpha_{2} \beta_{3} \gamma_{0}+\alpha_{2} \beta_{0} \gamma_{3}+\alpha_{0} \beta_{2} \gamma_{3}=0$;

(7) $\quad \alpha_{7} \beta_{0} \gamma_{0}+\alpha_{0} \beta_{7} \gamma_{0}+\alpha_{0} \beta_{0} \gamma_{7}+\alpha_{3} \beta_{1} \gamma_{0}+\alpha_{3} \beta_{0} \gamma_{1}+\alpha_{0} \beta_{3} \gamma_{1}=0$;

(8) $\quad \alpha_{8} \beta_{0} \gamma_{0}+\alpha_{0} \beta_{8} \gamma_{0}+\alpha_{0} \beta_{0} \gamma_{8}+\alpha_{3} \beta_{2} \gamma_{0}+\alpha_{3} \beta_{0} \gamma_{2}+\alpha_{0} \beta_{3} \gamma_{2}=0$;

$$
\begin{aligned}
& \vdots \\
& \alpha_{11} \beta_{0} \gamma_{0}+\alpha_{0} \beta_{11} \gamma_{0}+\alpha_{0} \beta_{0} \gamma_{11}+\alpha_{8} \beta_{1} \gamma_{0}+\alpha_{8} \beta_{0} \gamma_{1}+\alpha_{0} \beta_{8} \gamma_{1} \\
& +\alpha_{3} \beta_{5} \gamma_{0}+\alpha_{3} \beta_{0} \gamma_{5}+\alpha_{0} \beta_{3} \gamma_{5}+\alpha_{3} \beta_{2} \gamma_{1}+\alpha_{2} \beta_{4} \gamma_{0}+\alpha_{2} \beta_{0} \gamma_{4} \\
& +\alpha_{0} \beta_{2} \gamma_{4}+\alpha_{5} \beta_{3} \gamma_{0}+\alpha_{5} \beta_{0} \gamma_{3}+\alpha_{0} \beta_{5} \gamma_{3} \\
& +\alpha_{2} \beta_{1} \gamma_{3}=0
\end{aligned}
$$

And the center of $R$ is $\left\{k_{0}+k_{5} b a+k_{9} a c b+k_{10} b c a+k_{11} c b a \mid k_{i} \in K\right\}$ obviously. From (0), (1), (2) and (3), fhg $=k_{4} a c+k_{5} b a+k_{6} b c+k_{7} c a+k_{8} c b+$ $k_{9} a c b+k_{10} b c a+k_{11} c b a$ where $k_{4}=\alpha_{4} \gamma_{0} \beta_{0}+\alpha_{0} \gamma_{4} \beta_{0}+\alpha_{0} \gamma_{0} \beta_{4}+\alpha_{1} \gamma_{3} \beta_{0}+$ $\alpha_{1} \gamma_{0} \beta_{3}+\alpha_{0} \gamma_{1} \beta_{3}, k_{6}=\alpha_{6} \gamma_{0} \beta_{0}+\alpha_{0} \gamma_{6} \beta_{0}+\alpha_{0} \gamma_{0} \beta_{6}+\alpha_{2} \gamma_{3} \beta_{0}+\alpha_{2} \gamma_{0} \beta_{3}+\alpha_{0} \gamma_{2} \beta_{3}$, $k_{7}=\alpha_{7} \gamma_{0} \beta_{0}+\alpha_{0} \gamma_{7} \beta_{0}+\alpha_{0} \gamma_{0} \beta_{7}+\alpha_{3} \gamma_{1} \beta_{0}+\alpha_{3} \gamma_{0} \beta_{1}+\alpha_{0} \gamma_{3} \beta_{1}, k_{8}=\alpha_{8} \gamma_{0} \beta_{0}+$ $\alpha_{0} \gamma_{8} \beta_{0}+\alpha_{0} \gamma_{0} \beta_{8}+\alpha_{3} \gamma_{2} \beta_{0}+\alpha_{3} \gamma_{0} \beta_{2}+\alpha_{0} \gamma_{3} \beta_{2}$. From (0), $\alpha_{0}=0$ or $\beta_{0}=0$ or $\gamma_{0}=0$.

Case 1 . If $\alpha_{0}=0$, then $k_{4}=k_{6}=k_{7}=k_{8}=0$ by (4), (6), (7) and (8). So $f h g \in Z(R)$.

Case 2. If $\alpha_{0} \neq 0, \beta_{0}=\gamma_{0}=0$, then $\beta_{1} \gamma_{3}=\beta_{2} \gamma_{3}=\beta_{3} \gamma_{1}=\beta_{3} \gamma_{2}=0$ by (4), (6), (7) and (8). So $k_{4}=k_{6}=k_{7}=k_{8}=0$ and hence fhg $\in Z(R)$.

Case 3. If $\alpha_{0} \neq 0, \beta_{0} \neq 0$ and $\gamma_{0}=0$ or $\alpha_{0} \neq 0, \gamma_{0} \neq 0$ and $\beta_{0}=0$, then by $(0) \sim(11), h=0$ or $g=0$, respectively. So $f h g=0 \in Z(R)$.

Thus $R$ is cental right symmetric, so $R$ is central reversible though but IFP.

Example 2.6. We refer the argument in by [5, Example 4.10]. Let $K$ be a field. Then $R=K\langle a, b \mid a b=0\rangle$ is IFP by [5, Example 4.10]. However $b a=0$ and $(b a) a \neq 0=a(b a)$, entailing $b a \notin Z(R)$; hence $R$ is not central reversible. 
Following Agayev et al. [2], a ring $R$ is called central semicommutative if for any $a, b \in R, a b=0$ implies $a r b$ is a central element of $R$ for each $r \in R$. It is clear that every IFP ring is central semicommutative. By [2, Lemma 2.6] central semicommutative rings are Abelian and also by [14, Proposition 2.16] every left central symmetric ring is Abelian. In the following we prove that central reversible rings are Abelian.

Lemma 2.7. If a ring $R$ is central reversible, then $R$ is Abelian.

Proof. Let $e^{2}=e \in R, r \in R$. Then we get the following computation.

$e(1-e) r=0 \Rightarrow(1-e) r e \in Z(R) \Rightarrow 0=e(1-e) r e=(1-e) r e^{2}=(1-e) r e ;$

$(1-e) e r=0 \Rightarrow \operatorname{er}(1-e) \in Z(R) \Rightarrow 0=(1-e) \operatorname{er}(1-e)=\operatorname{er}(1-e)^{2}=\operatorname{er}(1-e)$. Thus we get $e r=e r e=r e$. So $R$ is Abelian.

The following example shows that Abelian rings need not be central reversible. This implies that the converse of Lemma 2.7 need not hold.

Example 2.8. Let $\mathbb{Z}$ be the ring of integers and let

$$
R=\left\{\left(\begin{array}{ll}
a & c \\
0 & b
\end{array}\right) \mid a-b \equiv c \equiv 0(\bmod 2)\right\} .
$$

Then, by [15, Example 13], $R$ is Abelian. Since $\left(\begin{array}{ll}0 & 2 \\ 0 & 0\end{array}\right)\left(\begin{array}{ll}2 & 0 \\ 0 & 0\end{array}\right)=0$ and $\left(\begin{array}{ll}2 & 0 \\ 0 & 0\end{array}\right)\left(\begin{array}{ll}0 & 2 \\ 0 & 0\end{array}\right)=$ $\left(\begin{array}{ll}0 & 4 \\ 0 & 0\end{array}\right) \notin Z(R), R$ is not central reversible.

By a simple computation we can prove that central left or right symmetric rings are central semicommutative.

Lemma 2.9. If a ring $R$ is central right (resp., left) symmertic, then $R$ is central semicommutative.

Proof. First suppose that $R$ is central right symmertic and let $a b=0 \in R$. Then $a b r=0$ implies $a r b \in Z(R)$ for all $r \in R$ since $R$ is central right symmertic. And suppose that $R$ is central left symmertic and let $a b=0 \in R$. Then $r a b=0$ implies $a r b \in Z(R)$ for all $r \in R$ since $R$ is central left symmertic.

In Example 2.6 $R$ is IFP but not central reversible and hence there exists a central semicommutative ring which is not a central right (resp., left) symmertic rings. But we do not answer whether central reversible rings are central semicommutative. So we remain this note by raising the following question.

Question. Are central reversible rings central semicommutative?

We will use the following terminology and notation for adjunction of 1 , due to Dorroh [9]. If $T$ is a ring without identity, its Dorroh extension is $T^{\prime}=\mathbb{Z} \oplus T$ (as additive groups) with multiplication defined by $\left(n_{1}, t_{1}\right)\left(n_{2}, t_{2}\right)=$ $\left(n_{1} n_{2}, t_{1} t_{2}+n_{1} t_{2}+n_{2} t_{1}\right)$. The property that $T$ be reduced (i.e., contain no nonzero nilpotent elements) is clearly preserved by Dorroh extensions. But, by [18, Example 2], neither symmetric nor the IFP condition is preserved by 
Dorroh extensions. So we may suspect that if $T$ is central right (resp., left) symmetric, then the Dorroh extension $T^{\prime}=\mathbb{Z} \oplus T$ is central right (resp., left) symmetric. But the following example eliminates the possibility.

Example 2.10. Let $T$ be as in Example 2.21 In the Dorroh extension $T^{\prime}=$ $\mathbb{Z} \oplus T$, we have $\alpha \beta \gamma=(1, a)(0, a)(0, a+b)=(0,0)$ and $\alpha \gamma \beta=(1, a)(0, a+$ $b)(0, a)=(0, a+b) \notin Z\left(T^{\prime}\right)$. Thus $T^{\prime}$ is not right central symmetric.

The following result is a direct consequence of simple computations.

Lemma 2.11. The classes of central reversible and central right (resp.,left) symmetric rings are closed under subrings and direct products.

Next one may suspect that a ring $R$ is central reversible if and only if $R[x]$ is cenrtal reversible. However the following example erases the possibility.

Example 2.12. We refer the argument in by [16, Example 2.1] and [12, Example 2]. Let $\mathbb{Z}_{2}$ be the field of integers modulo 2 and $A=\mathbb{Z}_{2}\left[a_{0}, a_{1}, a_{2}, b_{0}, b_{1}, b_{2}, c\right]$ be the free algebra of polynomials with zero constant terms in noncommuting indeterminates $a_{0}, a_{1}, a_{2}, b_{0}, b_{1}, b_{2}, c$ over $\mathbb{Z}_{2}$. Note that $A$ is a ring without identity and consider an ideal of the $\operatorname{ring} \mathbb{Z}_{2}+A$, say $I$, generated by

$$
\begin{gathered}
a_{0} b_{0}, a_{0} b_{1}+a_{1} b_{0}, a_{0} b_{2}+a_{1} b_{1}+a_{2} b_{0}, a_{1} b_{2}+a_{2} b_{1}, a_{2} b_{2}, a_{0} r b_{0}, a_{2} r b_{2}, \\
b_{0} a_{0}, b_{0} a_{1}+b_{1} a_{0}, b_{0} a_{2}+b_{1} a_{1}+b_{2} a_{0}, b_{1} a_{2}+b_{2} a_{1}, b_{2} a_{2}, b_{0} r a_{0}, b_{2} r a_{2}, \\
\left(a_{0}+a_{1}+a_{2}\right) r\left(b_{0}+b_{1}+b_{2}\right),\left(b_{0}+b_{1}+b_{2}\right) r\left(a_{0}+a_{1}+a_{2}\right), \text { and } r_{1} r_{2} r_{3} r_{4} r_{5},
\end{gathered}
$$

where $r, r_{1}, r_{2}, r_{3}, r_{4}, r_{5} \in A$. Then clearly $A^{5} \in I$. Next let $R=\left(\mathbb{Z}_{2}+A\right) / I$ and consider $R[x] \cong\left(\mathbb{Z}_{2}+A\right)[x] / I[x]$. Notice that $\left(c a_{0}+c a_{1} x+c a_{2} x^{2}\right)\left(b_{0}+\right.$ $\left.b_{1} x+b_{2} x^{2}\right) \in I[x]$, but $\left(b_{0}+b_{1} x+b_{2} x^{2}\right)\left(c a_{0}+c a_{1} x+c a_{2} x^{2}\right) \notin Z(R[x])$ because $b_{0} c a_{1}+b_{1} c a_{0}$ is not central; hence $R[x]$ is not central reversible.

Next we show that $R$ is central reversible. We call each product of the indeterminates $a_{0}, a_{1}, a_{2}, b_{0}, b_{1}, b_{2}, c$ a monomial and say that $\alpha$ is a monomial of degree $n$ if it is a product of exactly $n$ number of indeterminates. Let $H_{n}$ be the set of all linear combinations of monomials of degree $n$ over $\mathbb{Z}_{2}$.

Now set $f g \in I$ with $f, g \in R$, to see that $R$ is central reversible. We may write $f=\alpha+f_{1}+f_{2}+f_{3}+f_{4}+f_{5}, g=\beta+g_{1}+g_{2}+g_{3}+g_{4}+g_{5}$ for $\alpha, \beta \in \mathbb{Z}_{2}$, $f_{1}, g_{1} \in H_{1}, f_{2}, g_{2} \in H_{2}, f_{3}, g_{3} \in H_{3}, f_{4}, g_{4} \in H_{4}$ and $f_{5}, g_{5} \in I$ since $H_{i} \subseteq I$ for $i \geq 5$. Consider that $Z(R)=\left\{\gamma+h_{4}+h_{5} \mid \gamma \in \mathbb{Z}_{2}, h_{4} \in H_{4}, h_{5} \in I\right\}$ by the definition of $I$. Then we obtain $f g=\alpha \beta+\left(\alpha g_{1}+f_{1} \beta\right)+\left(\alpha g_{2}+f_{1} g_{1}+f_{2} \beta\right)+$ $\left(\alpha g_{3}+f_{1} g_{2}+f_{2} g_{1}+f_{3} \beta\right)+\left(\alpha g_{4}+f_{1} g_{3}+f_{2} g_{2}+f_{3} g_{1}+f_{4} \beta\right)+h \in I$ with $h \in I$, so $\alpha=0$ or $\beta=0$.

Case 1. If $\alpha=\beta=0$, then $f_{1} g_{1} \in I$ and $f_{1} g_{2}+f_{2} g_{1} \in I$. By Claim 1 and Claim 2 of [16, Example 2.1], we get $g_{1} f_{1} \in I$ and $g_{1} f_{2}+g_{2} f_{1} \in I$. Thus $g f \in Z(R)$.

Case 2. If $\alpha=0, \beta=1$ and $f_{1}=0$, then $f_{2} \in I$ and $f_{2} g_{1}+f_{3} \in I$; hence $f_{3} \in I$. Thus $g f \in Z(R)$. 
Case 3. If $\alpha=1, \beta=0$ and $g_{1}=0$, then $g_{2} \in I$ and $g_{3}+f_{1} g_{2} \in I$ : hence $g_{3} \in I$. Thus $g f \in Z(R)$.

So $g f \in Z(R)$ for each situation. Therefore $R$ is central reversible.

For any polynomial $f(x)$ in $R[x]$, let $C_{f(x)}$ denote the set of all coefficients of $f(x) . R$ is called Armendariz [20, Definition 1.1] if whenever any polynomials $f(x), g(x) \in R[x]$ satisfy $f(x) g(x)=0$, then $a b=0$ for each $a \in C_{f(x)}$ and $b \in C_{g(x)}$. Every reduced ring is Armendariz by Armendariz [6, Lemma 1].

Lemma 2.13 ([3, Proposition 1]). Suppose that $R$ is an Armendariz ring. If $f_{1}, \ldots, f_{n}$ are such that $f_{1} \cdots f_{n}=0$, then $a_{1} \cdots a_{n}=0$ where $a_{i}$ is a coefficient of $f_{i}$.

Proposition 2.14. Let $R$ be an Armendariz ring.

(1) $R$ is central reversible if and only if $R[x]$ is central reversible.

(2) $R$ is central right (resp., left) symmetric if and only if $R[x]$ is central (resp., left) symmetric.

Proof. It suffices to show the necessity.

(1) Assume that $R$ is central reversible. Let $f(x) g(x)=0$ for $f(x), g(x) \in$ $R[x]$. Then $a b=0$ for any $a \in C_{f(x)}, b \in C_{g(x)}$. Since $R$ is central reversible, $b a \in Z(R)$. This yields that $g(x) f(x) \in Z(R)$ and thus $R[x]$ is central reversible.

(2) Assume that $R$ is central symmetric. Let $f(x) g(x)=0$ for $f(x), g(x)$, $h(x) \in R[x]$. Then $a b c=0$ for any $a \in C_{f(x)}, b \in C_{g(x)}$ and $c \in C_{h(x)}$ by Lemma 2.13. Since $R$ is central right(resp., left) symmetric, $a c b \in Z(R)$ (resp., $b a c \in Z(R)$. This yields that $f(x) h(x) g(x) \in Z(R)$ (resp., $g(x) f(x) h(x)=0$ ) and thus $R[x]$ is central right (resp., left) symmetric.

This example also provides a counterexample to a conjecture that if a ring $R$ is central reversible, then $R / I$ is also central reversible for any ideal $I$ in $R$. In Example 2.12, $\left(\mathbb{Z}_{2}+A\right)[x]$ is a domain so central reversible clearly, but the factor ring $\left(\mathbb{Z}_{2}+A\right)[x] / I[x] \cong R[x]$ is not central reversible.

Proposition 2.15. (1) Let $R$ be a ring. Then $e R$ and $(1-e) R$ are central reversible for some central idempotent $e$ of $R$ if and only if $R$ is central reversible.

(2) Let $R$ be a ring and $\Delta$ be a multiplicatively closed subset of $R$ consisting of central regular elements. Then $R$ is central reversible if and only if $\Delta^{-1} R$ is central reversible.

(3) Let $R$ be a ring. Then $e R$ and $(1-e) R$ (resp., Re and $R(1-e)$ ) are central right (resp., left) symmetric for some central idempotent $e$ of $R$ if and only if $R$ is central right (resp., left) symmetric.

(4) Let $R$ be a ring and $\Delta$ be a multiplicatively closed subset of $R$ consisting of central regular elements. Then $R$ is central right (resp., left) symmetric if and only if $\Delta^{-1} R$ (resp., $R \Delta^{-1}$ is central right (resp., left) symmetric.

Proof. For the proofs (1), (2), (3) and (4), it suffices to show that the necessities by Lemma 2.11 . 
(1) Let $a b=0$ for $a, b \in R$. Then $e a b=0$ and $(1-e) a b=0$. So we have $e b a \in Z(e R)$ and $(1-e) b a \in Z((1-e) R)$ by hypothesis. Hence, for any $r \in R$, ebaer $=e r e b a$ and $(1-e) b a(1-e) r=(1-e) r(1-e) b a$ and so bar $=r b a$. Thus $b a \in Z(R)$ and therefore $R$ is central reversible.

(2) Let $\alpha \beta=0$ with $\alpha=u^{-1} a, \beta=v^{-1} b, u, v \in \Delta$ and $a, b \in R$. Since $\Delta$ is contained in the center of $R$, we have $0=\alpha \beta=\left(u^{-1} a\right)\left(v^{-1} b\right)=u^{-1} v^{-1} a b$ and $a b=0$. But $R$ is central reversible by supposition, so $b a \in Z(R)$ and we have $\beta \alpha=\left(v^{-1} b\right)\left(u^{-1} a\right)=v^{-1} u^{-1} b a \in Z\left(\Delta^{-1} R\right)$; hence $\Delta^{-1} R$ is central reversible.

(3) Let $a b c=0$ for $a, b, c \in R$. Then $e a b c=0$ and $(1-e) a b c=0$. So we have $e a c b \in Z(e R)$ and $(1-e) a c b \in Z((1-e) R)$ by hypothesis. Hence, for any $r \in R$, eacber $=$ ereacb and $(1-e) a c b(1-e) r=(1-e) r(1-e) a c b$ and so $a c b r=r a c b$. Thus $a c b \in Z(R)$ and therefore $R$ is right central symmetric. And the proof of left vision is similar to the right vision.

(4) The proof of left version by [14, Proposition 2.25]. And the proof of right version is similar to the left version.

The ring of Laurent polynomials in $x$, coefficients in a ring $R$, consists of all formal sums $\sum_{i=k}^{n} m_{i} x^{i}$ with obvious addition and multiplication, where $m_{i} \in R$ and $k, n$ are (possibly negative) integers; denote it by $R\left[x ; x^{-1}\right]$.

Lemma 2.16. For a ring $R, R[x]$ is central reversible (resp., central right (resp., left) symmetric) if and only if $R\left[x ; x^{-1}\right]$ is central reversible (resp., central right (resp., left) symmetric).

Proof. For the proof, it suffices to show the necessity by Lemma 2.11. Let $\Delta=\left\{1, x, x^{2}, \ldots\right\}$ then clearly $\Delta$ is a multiplicatively closed subset of $R[x]$ consisting of central regular elements. Since $R\left[x ; x^{-1}\right]=\Delta^{-1} R[x]$, it follows that $R\left[x ; x^{-1}\right]$ is central reversible by Proposition $2.15(3)$.

Proposition 2.17. Let $R$ be a ring and suppose that $Z(R)$ contains an infinite subring whose nonzero elements are regular in $R$. Then the following statements are equivalent:

(1) $R$ is central reversible (resp., right central symmetric).

(2) $R[x]$ is central reversible (resp., right central symmetric).

(3) $R\left[x ; x^{-1}\right]$ is central reversible (resp., right central symmetric).

Proof. By Lemma 2.11 and Lemma 2.16, it suffices to show $(1) \Rightarrow(2)$. It is well-known that $R[x]$ is a subdirect product of $R$ 's, under given conditions. Thus $R[x]$ is central reversible by Lemma 2.11 .

Proposition 2.18. Given an Armendariz ring $R$, the following conditions are equivalent:

(1) $R$ is central reversible;

(2) $R[x]$ is central reversible;

(3) $R\left[x ; x^{-1}\right]$ is central reversible. 
Proof. By Lemma 2.11 and Lemma 2.16, it suffices to show (1) $\Rightarrow(2)$. Let $f=\sum_{i=0}^{m} a_{i} x^{i}, g=\sum_{j=0}^{n} b_{j} x^{j}$ be the polynomials in $R[x]$ such that $f g=0$. Then since $R$ is Armendariz, each $a_{i} b_{j}=0$; but $R$ is central reversible so $b_{j} a_{i} \in Z(R)$ for all $i, j$. Consequently we obtain $g f \in Z(R[x])$ and $R[x]$ is central reversible.

Proposition 2.19. Given an Armendariz ring $R$, the following conditions are equivalent:

(1) $R$ is central right (resp., left) symmetric;

(2) $R[x]$ is central right (resp., left) symmetric;

(3) $R\left[x ; x^{-1}\right]$ is central right (resp., left) symmetric.

Proof. By Lemma 2.11 and Lemma 2.16, it suffices to show (1) $\Rightarrow(2)$. Let $f=\sum_{i=0}^{m} a_{i} x^{i}, g=\sum_{j=0}^{n} b_{j} x^{j}$ and $h=\sum_{k=0}^{l} c_{k} x^{k}$ be the polynomials in $R[x]$ such that $f g h=0$. Then since $R$ is Armendariz, each $a_{i} b_{j} c_{k}=0$; but $R$ is right central symmetric so $a_{i} c_{k} b_{j} \in Z(R)$ for all $i, j, k$. Consequently we obtain $f h g \in Z(R[x])$ and $R[x]$ is right central symmetric.

A ring $R$ is usually called (von Neumann) regular if for each $a \in R$ there exists $b \in R$ such that $a=a b a$.

Proposition 2.20. Given a regular ring $R$, the following conditions are equivalent:

(1) $R$ is reduced;

(2) $R$ is symmetric;

(3) $R$ is reversible;

(4) $R$ is IFP;

(5) $R$ is Abelian;

(6) $R$ is central reversible;

(7) $R$ is central right (resp., left) symmetric.

Proof. The equivalence relation from (1) to (5) are shown by [10, Thoerem 3.2]. $(3) \Rightarrow(6),(2) \Rightarrow(7)$ and $(7) \Rightarrow(6)$ are obtained from definitions. And $(6) \Rightarrow(5)$ is true by Lemma 2.7 .

But, for rings without identity, these are no longer true, as illustrated by the following examples.

Example 2.21. Let $S=\{a, b\}$ be the semigroup with multiplication $a^{2}=$ $a b=a, b^{2}=b a=b$. Put $T=\mathbb{F}_{2} S$, which is a four-element semigroup ring without identity. Then $T$ is symmetric but not reversible by [18, Example 1]. Moreover $Z(T)=\{0\}$ and hence $T$ is also right central symmetric. And $a(a+b)=0$ but $(a+b) a=a+b \notin Z(T)$. So $T$ is not central reversible.

Let $R$ be a ring and $n$ be a positive integer. Following the literature, define $N_{n}(R)=\left\{A \in U_{n}(R) \mid\right.$ each diagonal entry of $A$ is zero $\}$. 
Example 2.22. Let $R$ be any ring. $N_{k}(R)$ is both left and right (central) symmetric for all $k=2,3$. For, $A B C=0$ for all $A, B, C \in N_{k}(R)$ when $k=2$ or $k=3$.

$N_{4}(R)$ is also both left and right (central) symmetric. For, $A B C=\alpha e_{14}$ (with $\alpha \in R$ ) for all $A, B, C \in N_{4}(R)$, and so $(A B C) D=0=D(A B C)$ for all $D \in N_{4}(R)$.

However $N_{j}(R)$ is neither left nor right central symmetric for $j \geq 5$. For, $e_{23} e_{12} e_{34}=0$ and $e_{12} e_{34} e_{23}=0$, but $e_{12} e_{23} e_{34}=e_{14}$ is not central in $N_{j}(R)$ as can be seen by $e_{14} e_{45}=e_{15}$ and $e_{45} e_{14}=0$.

Example 2.23. Let $R$ be any ring. Note that $\left(\begin{array}{lll}0 & 0 & 0 \\ 0 & 0 & 1 \\ 0 & 0 & 0\end{array}\right)\left(\begin{array}{lll}0 & 1 & 0 \\ 0 & 0 & 0 \\ 0 & 0 & 0\end{array}\right)=0$. But we have $\left(\begin{array}{lll}0 & 1 & 0 \\ 0 & 0 & 0 \\ 0 & 0 & 0\end{array}\right)\left(\begin{array}{lll}0 & 0 & 0 \\ 0 & 0 & 1 \\ 0 & 0 & 0\end{array}\right) \neq 0$. So $N_{3}(R)$ is not reversible. But $N_{3}(R)$ is central reversible, since $A B C=0$ for all $A, B, C \in N_{3}(R)$.

Acknowledgments. The authors must thank the referee very much for very careful reading of the manuscript and many valuable suggestions that improved the paper by much. The second named author was supported by the Basic Science Research Program through the National Research Foundation of Korea(NRF) funded by the Ministry of Education(NRF-2013R1A1A4A01008 108), and the third named author was supported by Basic Science Research Program through the National Research Foundation of Korea(NRF) funded by the Ministry of Education(NRF-2013R1A1A2A10004687).

\section{References}

[1] N. Agayev, A. Harmanci, and S. Halicioglu, Extended Armendariz rings, Algebras Groups Geom. 26 (2009), no. 4, 343-354.

[2] N. Agayev, T. Ozen, and A. Harmanci, On a Class of semicommutative rings, Kyungpook Math. J. 51 (2011), no. 3, 283-291.

[3] D. D. Anderson and V. Camillo, Armendariz rings and gaussian rings, Comm. Algebra 26 (1998), no. 7, 2265-2272.

[4] - Semigroups and rings whose zero products commute, Comm. Algebra 27 (1999), no. $6,2847-2852$

[5] R. Antoine, Nilpotent elements and Armendariz rings, J. Algebra 319 (2008), no. 8, $3128-3140$.

[6] E. P. Armendariz, A note on extensions of Baer and P.P.-rings, J. Austral. Math. Soc. 18 (1974), 470-473.

[7] H. E. Bell, Near-rings in which each element is a power of itself, Bull. Austral. Math. Soc. 2 (1970), 363-368.

[8] P. M. Cohn, Reversible rings, Bull. London Math. Soc. 31 (1999), no. 6, 641-648.

[9] J. L. Dorroh, Concerning adjunctions to algebras, Bull. Amer. Math. Soc. 38 (1932), no. $2,85-88$.

[10] K. R. Goodearl, Von Neumann Regular Rings, Pitman, London, 1979.

[11] J. M. Habeb, A note on zero commutative and duo rings, Math. J. Okayama Univ. 32 (1990), 73-76.

[12] C. Huh, Y. Lee, and A. Smoktunowicz, Armendariz rings and semicommutative rings, Comm. Algebra 30 (2002), no. 2, 751-761.

[13] T. W. Hungerford, Algebra, Springer-Verlag, New York, 1974. 
[14] G. Kafkas, B. Ungor, S. Halicioglu, and A. Harmanci, Generalized symmetric rings, Algebra Discrete Math. 12 (2011), no. 2, 72-84.

[15] N. K. Kim and Y. Lee, Armendariz rings and reduced rings, J. Algebra 223 (2000), no. $2,477-488$.

[16] _ Extensions of reversible rings, J. Pure Appl. Algebra 185 (2003), no. 1-3, 207223

[17] J. Lambek, On the representation of modules by sheaves of factor modules, Canad. Math. Bull. 14 (1971), 359-368.

[18] G. Marks, Reversible and symmetric rings, J. Pure Appl. Algebra 174 (2002), no. 3, 311-318.

[19] G. Mason, Reflexive ideals, Comm. Algebra 9 (1981), no. 17, 1709-1724.

[20] M. B. Rege and S. Chhawchharia, Armendariz rings, Proc. Japan Acad. Ser. A Math. Sci. 73 (1997), no. 1, 14-17.

[21] G. Shin, Prime ideals and sheaf representation of a pseudo symmetric rings, Trans. Amer. Math. Soc. 184 (1973), 43-60.

[22] A. A. Tuganbaev, Semidistributive Modules and Rings, Mathematics and its Applications 449, Kluwer Academic Publishers, Dordrecht, 1998.

Da Woon Jung

Department of Mathematics

Pusan National University

Pusan 609-735, Korea

E-mail address: jungdw@pusan.ac.kr

NAM KYUn Kim

College of Liberal Arts and Sciences

Hanbat National University

DAEJEON 305-719, KoREA

E-mail address: nkkim@hanbat.ac.kr

YANG LEE

Department of Mathematics Education

Pusan National University

PUSAN 609-735, Korea

E-mail address: ylee@pusan.ac.kr

Sung Ju RYu

Department of Mathematics

Pusan National University

Pusan 609-735, Korea

E-mail address: sjryu@pusan.ac.kr 